\title{
On computing the zeros of periodic systems
}

\author{
Andras Varga \\ German Aerospace Center \\ DLR - Oberpfaffenhofen \\ Institute of Robotics and System Dynamics \\ D-82234 Wessling, Germany \\ Andras.Varga@dlr.de
}

\author{
Paul Van Dooren \\ CESAME \\ Université catholique de Louvain \\ B-1348 Louvain-la-Neuve, Belgium \\ vDooren@csam.ucl.ac.be
}

\begin{abstract}
We present an efficient and numerically reliable approach to compute the zeros of a periodic system. The zeros are defined in terms of the transfer-function matrix corresponding to an equivalent lifted statespace representation as constant system. The proposed method performs locally row compressions of the associated system pencil to extract a low order pencil which contains the zeros (both finite and infinite) as well as the Kronecker structure of the periodic system. The proposed algorithm belongs to the family of fast, structure exploiting algorithms and relies exclusively on using orthogonal transformations. For the overall zeros computation a certain form of numerical stability can be ensured.
\end{abstract}

\section{Introduction}

Among the important open computational problems listed in a recent survey [23], the computation of zeros of the transfer-function matrix of a periodic system is one which has many useful applications. Besides characterizing when the system is minimum-phase or not, the zeros provide information on several structural properties of a system. A general, efficient and numerically reliable algorithm to compute the zeros of a periodic system can be seen as a universal tool for the analysis of periodic systems. For instance, reachability/stabilizability and observability/detectability can be easily studied by computing the zeros of particular periodic systems without outputs or inputs, respectively. Even the poles of a periodic system can be seen as a particular type of zeros for a system with no inputs and no outputs. A recent algorithm using this to evaluate the transfer-function matrix of a periodic system [22], relies on the computation of poles and zeros of particular single-input single-output periodic system.

We consider the periodic time-varying system of the form

$$
\begin{aligned}
x(k+1) & =A_{k} x(k)+B_{k} u(k) \\
y(k) & =C_{k} x(k)+D_{k} u(k)
\end{aligned}
$$

where the matrices $A_{k} \in \mathrm{R}^{n_{k+1} \times n_{k}}, B_{k} \in \mathrm{R}^{n_{k+1} \times m}$, $C_{k} \in \mathrm{R}^{p \times n_{k}}, D_{k} \in \mathrm{R}^{p \times m}$ are periodic with period $K \geq 1$. For the computation of zeros it is important to consider this more general case of time-varying state dimensions. Since the transmission zeros of a standard system are defined in term of a minimal realization, a similar definition is appropriate also for the zeros of periodic systems (see for example [14]). However, the minimal realization theory of these systems revealed (see for example $[5,8]$ ) that minimal order (i.e., reachable and observable) state-space realizations of periodic systems have, in general, time-varying state-space dimensions. Note that periodic systems with time-varying dimensions have been already considered earlier in [12, 9]. But only recently, numerically reliable algorithms for systems with time-varying dimensions have been developed. Notable examples are the recent algorithms for the computation minimal realizations [21] and the evaluation of the transfer-function matrix of periodic systems [22]. Finally, the development of general algorithms able to address the time-varying case, is one of the requirements formulated for a satisfactory numerical algorithm for periodic systems [23].

The definition of zeros of a periodic system can be introduced starting from any of the time-invariant lifted reformulations $[4,10]$. These zeros have a nice interpretation in terms of periodic blocking property of exponentially periodic input signals [2]. In this paper we use the lifting approach proposed by Meyer and Burrus in [15] as basis for the definition of zeros. However, the computation of zeros using this lifted reformulation by using standard methods (e.g., as those proposed in [6]), involves forming products of up to $K$ matrices. Apart from being computationally expensive, the explicit computation of the lifted reformulation can lead to severe numerical difficulties. A second lifting approach appears in the works of several authors $[17,7,12]$ and leads to a large order standard system representation with sparse and highly structured matrices. While the direct application of the numerically stable method of [16] is certainly possible, the computational complexity for large order systems is very high. Assuming constant dimensions $n_{i}=n$, such an algorithm has a com- 
plexity of $O\left((K n)^{3}\right)$, instead of an expected complexity of $O\left(K n^{3}\right)$ for a satisfactory algorithm [23].

Although the lifting techniques are useful for their theoretical insight, their sparsity and structure may not be suited for numerical computations. This is why, in parallel to the theoretical developments, numerical methods have been developed that try to exploit this structure. The purpose of this paper is to propose a numerical approach to compute the zeros of the transfer-function matrix (TFM) of the periodic system (1) which meets the requirements of generality, speed and accuracy for a good numerical algorithm for periodic systems as formulated in [23]. This goal is mainly achieved by exploiting the sparse structure of the associated system pencil by performing locally row compressions to extract a low order pencil (of order about $n=\max _{i}\left\{n_{i}\right\}$ ) which contains the zeros (both finite and infinite) as well as the Kronecker structure of the periodic system. For this pencil, standard methods can be employed to determine the zeros and the Kronecker structure (e.g., [16]). The new algorithm belongs to the family of fast, structure exploiting algorithms and relies exclusively on using orthogonal transformations. This is why, for the overall zeros computation a certain form of numerical stability can be ensured.

Notation. For a $K$-periodic matrix $X_{i}$ we use alternatively the script notation

$$
\mathcal{X}_{k}:=\operatorname{diag}\left(X_{k}, X_{k+1}, \ldots, X_{k+K-1}\right),
$$

which associates the block-diagonal matrix $\mathcal{X}_{k}$ to the cyclic matrix sequence $X_{i}, i=k, \ldots, k+K-1$ starting at time moment $k$. We reserve the script notation $\mathcal{X}$ (i.e., without subscript) for $\mathcal{X}=\mathcal{X}_{1}$. By using the script notation, the periodic system (1) will be alternatively denoted by the quadruple $\left(\mathcal{A}_{k}, \mathcal{B}_{k}, \mathcal{C}_{k}, \mathcal{D}_{k}\right)$ or $(\mathcal{A}, \mathcal{B}, \mathcal{C}, \mathcal{D})$ if $k=1$. The transition matrix of the system (1) is defined by the $n_{j} \times n_{i}$ matrix $\Phi_{A}(j, i)=A_{j-1} A_{j-2} \cdots A_{i}$, where $\Phi_{A}(i, i):=I_{n_{i}}$. The state transition matrix over one period $\Phi_{A}(j+K, j) \in \mathrm{R}^{n_{j} \times n_{j}}$ is called the monodromy matrix of system (1) at time $j$ and its eigenvalues are called characteristic multipliers at time $j$.

\section{Zeros and poles of periodic systems}

To define the zeros and poles of the periodic system (1), we define first the transfer-function matrix (TFM) corresponding to the associated lifted system introduced in [15]. This lifting technique uses the input-output behavior of the system over time intervals of length $K$, rather then 1 . For a given sampling time $k$, the corresponding $m K$-dimensional input and $p K$-dimensional output vectors are

$$
\begin{aligned}
& u_{k}^{L}(h)=\left[u^{T}(k+h K) \cdots u^{T}(k+h K+K-1)\right]^{T} \\
& y_{k}^{L}(h)=\left[y^{T}(k+h K) \cdots y^{T}(k+h K+K-1)\right]^{T}
\end{aligned}
$$

and an $n_{k}$-dimensional state vector is defined as

$$
x_{k}^{L}(h):=x(k+h K)
$$

The lifted system has the form

$$
\begin{aligned}
x_{k}^{L}(h+1) & =F_{k}^{L} x_{k}^{L}(h)+G_{k}^{L} u_{k}^{L}(h) \\
y_{k}^{L}(h) & =H_{k}^{L} x_{k}^{L}(h)+L_{k}^{L} u_{k}^{L}(h)
\end{aligned}
$$

where

$$
\begin{aligned}
& F_{k}^{L}=\Phi_{A}(k+K, k) \\
& G_{k}^{L}=\left[\Phi_{A}(k+K, k+1) B_{k} \cdots B_{k+K-1}\right] \\
& H_{k}^{L}=\left[\begin{array}{c}
C_{k} \\
C_{k+1} \Phi_{A}(k+1, k) \\
\vdots \\
C_{k+K-1} \Phi_{A}(k+K-1, k)
\end{array}\right] \\
& L_{k}^{L}=\left[\begin{array}{cccc}
D_{k} & 0 & \cdots & 0 \\
L_{k, 2,1} & D_{k+1} & \cdots & 0 \\
\vdots & \vdots & \ddots & \vdots \\
L_{k, K, 1} & L_{k, K, 2} & \cdots & D_{k+K-1}
\end{array}\right]
\end{aligned}
$$

with

$$
L_{k, i, j}=C_{k+i-1} \Phi_{A}(k+i-1, k+j) B_{k+j-1},
$$

for $i=2, \ldots, K, j=1,2, \ldots K-1$, and $i>j$.

The system (2) is called the standard lifted system at time $k$ of the given $K$-periodic system (1). The associated $\mathrm{TFM} W_{k}(z)$ is

$$
W_{k}^{L}(z)=H_{k}^{L}\left(z I_{n_{k}}-F_{k}^{L}\right)^{-1} G_{k}^{L}+L_{k}^{L}
$$

and depends on the sampling time $k$. Obviously $W_{k+K}^{L}(z)=W_{k}^{L}(z)$ and the TFMs at two successive values of $k$ are related by the following relation [10]

$$
W_{k+1}^{L}(z)=\left[\begin{array}{cc}
0 & I_{p(K-1)} \\
z I_{p} & 0
\end{array}\right] W_{k}^{L}(z)\left[\begin{array}{cc}
0 & z^{-1} I_{m} \\
I_{m(K-1)} & 0
\end{array}\right]
$$

To define the zeros and poles of a periodic system we assume in what follows that the given periodic system (1) is minimal (i.e., completely reachable and completely observable). It follows (see e.g., [2]) that the lifted system (2) is minimal too and the converse is also true.

Definition 1. The zeros of the periodic system $\left(\mathcal{A}_{k}, \mathcal{B}_{k}, \mathcal{C}_{k}, \mathcal{D}_{k}\right)$ are the invariant zeros (see [14]) of the associated lifted system (2) or, equivalently, the transmission zeros of the associated TFM $W_{k}(z)(3)$.

Definition 2. The poles of the periodic system $\left(\mathcal{A}_{k}, \mathcal{B}_{k}, \mathcal{C}_{k}, \mathcal{D}_{k}\right)$ are the eigenvalues of the monodromy matrix $F_{k}^{L}$, or equivalently, the poles of the associated TFM $W_{k}(z)(3)$. 
The invariant zeros of the lifted system (2) are the Smith-zeros of the associated system pencil

$$
S_{k}^{L}(z)=\left[\begin{array}{cc}
F_{k}^{L}-z I_{n_{k}} & G_{k}^{L} \\
H_{k}^{L} & L_{k}^{L}
\end{array}\right]
$$

Using this pencil, we can extend the standard definitions of zeros at infinity, as well as of the system pencil Kronecker structure [16] to periodic systems. However, since the pencil (4) involves forming of matrix products, this pencil is certainly not suited for the reliable numerical computation of zeros. We will use instead a more convenient representation, the so-called stacked lifted representation [12], which is a time-invariant descriptor system representation of the form

$$
\begin{aligned}
E_{k}^{S} x_{k}^{S}(h+1) & =F_{k}^{S} x_{k}^{S}(h)+G_{k}^{S} u_{k}^{L}(h) \\
y_{k}^{L}(h) & =H_{k}^{S} x_{k}^{S}(h)+L_{k}^{S} u_{k}^{L}(h)
\end{aligned}
$$

where $G_{k}^{S}=\mathcal{B}_{k}, H_{k}^{S}=\mathcal{C}_{k}, L_{k}^{S}=\mathcal{D}_{k}$, and

$$
F_{k}^{S}-z E_{k}^{S}=\left[\begin{array}{ccccc}
A_{k} & -I_{n_{k+1}} & O & \cdots & O \\
O & \ddots & \ddots & \ddots & \vdots \\
\vdots & \ddots & \ddots & -I_{n_{k+K-2}} & O \\
O & & \ddots & A_{k+K-2} & -I_{n_{k+K-1}} \\
-z I_{n_{k}} & O & \cdots & O & A_{k+K-1}
\end{array}\right]
$$

The TFM of the stacked lifted system is

$$
W_{k}^{S}(z)=H_{k}^{S}\left(z E_{k}^{S}-F_{k}^{S}\right)^{-1} G_{k}^{S}+L_{k}^{S}
$$

and the associated system pencil is defined as

$$
S_{k}^{S}(z)=\left[\begin{array}{cc}
F_{k}^{S}-z E_{k}^{S} & G_{k}^{S} \\
H_{k}^{S} & L_{k}^{S}
\end{array}\right] .
$$

The relation of these matrices with those of the standard lifted system is explained by the following lemma, which is easily proven by standard elimination.

Lemma 1 There exist invertible matrices $T_{l}$ and $T_{r}$ and matrices $X$ and $Y$ such that:

$$
\begin{gathered}
{\left[\begin{array}{cc|c}
I_{\mu_{k}} & O & O \\
O & F_{k}^{L}-z I_{n_{k}} & G_{k}^{L} \\
\hline O & H_{k}^{L} & L_{k}^{L}
\end{array}\right]=} \\
{\left[\begin{array}{c|c}
T_{l} & O \\
\hline X & I
\end{array}\right] \cdot\left[\begin{array}{c|c}
F_{k}^{S}-z E_{k}^{S} & G_{k}^{S} \\
\hline H_{k}^{S} & L_{k}^{S}
\end{array}\right] \cdot\left[\begin{array}{c|c}
T_{r} & Y \\
\hline O & I
\end{array}\right],}
\end{gathered}
$$

where $\mu_{k}:=n_{k+1}+\cdots+n_{k+K-1}$.

By Schur complementation, it is now easy to see that $W_{k}^{S}(z)=W_{k}^{L}(z)$, that is, the TFMs of the stacked and standard lifted systems are the same. Moreover, both system pencils are essentially equivalent since the transformations of the above lemma only eliminated the nondynamical part of the systems matrix $S_{k}^{S}(z)$. Thus, we have the following immediate results which underly the proposed computational algorithm in the next section:
Lemma 2 The zeros of the periodic system $\left(\mathcal{A}_{k}, \mathcal{B}_{k}, \mathcal{C}_{k}, \mathcal{D}_{k}\right)$ are the invariant zeros of the stacked lifted system (5), and hence the zeros of the associated system pencil (7).

Lemma 3 The poles of the periodic system $\left(\mathcal{A}_{k}, \mathcal{B}_{k}, \mathcal{C}_{k}, \mathcal{D}_{k}\right)$ are the finite generalized eigenvalues of the pencil $F_{k}^{S}-z E_{k}^{S}$ defined in (6).

\section{Computational approach}

In this section we propose an efficient computational approach to determine the zeros at $k=1$ of the standard lifted system (2). The zeros for other time moments $k=2, \ldots, K$ can be similarly computed by just permuting the order of the underlying matrices. To simplify the notation for the case $k=1$, we drop in what follows the index used for the sampling time in the lifted system matrices. Before starting our developments, we discuss shortly possible approaches relying on existing algorithms for standard systems.

A straightforward approach to compute the zeros of the $p K \times m K$ TFM $W(z)$ is to apply the algorithm of [6] to the system matrix (4) and to extract additional information on zeros and Kronecker structure using the results of [19]. However, because the construction of the standard lifted system involves matrix multiplications, this approach is certainly not recommendable for numerical computations. To avoid matrix multiplications, we can employ the general approach of [16] to the stacked lifted system and compute the system zeros as the invariant zeros of the system matrix (7). This approach is numerically reliable because it relies exclusively on using orthogonal transformations. But since it ignores the structure of the problem, the computational complexity of this approach is too high. To compute the zeros, the computational complexity is, in the worst case, of the order of $O((\mu+K p)(\mu+K m) \mu)$ operations, where $\mu=\sum_{i=1}^{K} n_{i}$. For example, in the case of a periodic system with constant state dimensions $n_{i}=n$, the computational complexity is $O\left(K^{3}(n+p)(n+m) n\right)$ instead of a desirable complexity of $O(K(n+p)(n+m) n)$ as formulated in [23] for a satisfactory algorithm for periodic systems. In what follows, we show that such a computational complexity can be indeed achieved by exploiting the problem structure.

The idea of fast numerical algorithms to compute eigenvalues of products of square matrices has been introduced by Van Dooren [20]. The algorithm proposed in [20], can be used to compute the poles of periodic systems with constant dimensions by deflating the $(K-1) n$ simple eigenvalues at infinity of the pencil $F^{S}-z E^{S}$ by applying $(K-1)$ orthogonal transformations on low order submatrices of this pencil. This approach is an 
orthogonal version of the technique employed by Luenberger [13] and is equivalent to the "swapping" technique described in [1]. The extension of this algorithm to the time-varying case is relatively straightforward and is a particular case of the approach proposed in this paper. In what follows we show how this idea can be applied to deflate a part of the system pencil (7) which corresponds to $\sum_{i=2}^{K} n_{i}$ simple eigenvalues at infinity. Since the multiplicity of eigenvalues are by definition in excess one with respect to the multiplicity of infinite zeros, this deflation will not affect the computation of both finite and infinite zeros.

Consider instead $S^{S}(z)$ in (7), an equivalent pencil $S(z)$ with permuted block rows and columns

$$
S(z)=\left[\begin{array}{ccccc}
S_{1} & -T_{1} & O & \cdots & O \\
O & S_{2} & -T_{2} & \cdots & O \\
\vdots & \ddots & \ddots & \ddots & \vdots \\
O & & & S_{K-1} & -T_{K-1} \\
-z T_{K} & O & \cdots & O & S_{K}
\end{array}\right]
$$

where for $i=1, \ldots, K$

$$
S_{i}:=\left[\begin{array}{cc}
A_{i} & B_{i} \\
C_{i} & D_{i}
\end{array}\right], \quad T_{i}:=\left[\begin{array}{cc}
I_{n_{i+1}} & O \\
O & O
\end{array}\right] .
$$

Consider the $\left(n_{2}+n_{3}+2 p\right)$-th order orthogonal transformation matrix $U_{1}$ compressing the rows of the $\left(n_{2}+\right.$ $\left.n_{3}+2 p\right) \times\left(n_{3}+m\right)$ matrix $\left[\begin{array}{c}-T_{1} \\ S_{2}\end{array}\right]$ to $\left[\begin{array}{c}R_{1} \\ O\end{array}\right]$, where $R_{1}$ is an $r_{2} \times\left(n_{3}+m\right)$ full row rank matrix with $n_{2} \leq r_{2} \leq n_{2}+\min (p, m)$. Applying $U_{1}$ to the first two blocks rows of $S(z)$ we obtain for the nonzero elements

$$
U_{1}\left[\begin{array}{ccc}
S_{1} & -T_{1} & O \\
O & S_{2} & -T_{2}
\end{array}\right]=\left[\begin{array}{ccc}
\widetilde{S}_{1} & R_{1} & -\widetilde{T}_{1} \\
\widehat{S}_{2} & O & -\widehat{T}_{2}
\end{array}\right]
$$

which defines the new matrices $\widehat{S}_{2}$ and $\widehat{T}_{2}$. Then construct the orthogonal transformations $U_{i}$ for $i=$ $2, \ldots K-1$ such that

$$
U_{i}\left[\begin{array}{ccc}
\widehat{S}_{i} & -\widehat{T}_{i} & O \\
O & S_{i+1} & -T_{i+1}
\end{array}\right]=\left[\begin{array}{ccc}
\widetilde{S}_{i} & R_{i} & -\widetilde{T}_{i} \\
\widehat{S}_{i+1} & O & -\widehat{T}_{i+1}
\end{array}\right]
$$

where $R_{i}$ are full row rank matrices. Applying the transformations $U_{i}$ successively to the $i$ th and $(i+1)$ th block rows of the pencil $S(z)$, we get a reduced pencil

$$
\left[\begin{array}{c|cccc}
\widetilde{S}_{1} & R_{1} & -\widetilde{T}_{1} & O & O \\
\widetilde{S}_{2} & O & R_{2} & \ddots & O \\
\vdots & \vdots & \vdots & \ddots & -\widetilde{T}_{K-2} \\
\widetilde{S}_{K-1}-z \widetilde{T}_{K-1} & O & O & \cdots & R_{K-1} \\
\hline \widehat{S}_{K}-z \widehat{T}_{K} & O & O & \cdots & O
\end{array}\right]
$$

which is orthogonally similar to the original system pencil $S^{S}(z)$ in (7). Since the matrices $R_{i}$ have full row rank, the subpencil

$$
\widehat{S}_{K}-z \widehat{T}_{K}
$$

will contain all finite zeros of the original pencil. The Kronecker structure and the infinite zeros of $S^{S}(z)$ are essentially those of the subpencil

$$
\left[\begin{array}{cc}
\widetilde{S}_{K-1}-z \widetilde{T}_{K-1} & R_{K-1} \\
\widehat{S}_{K}-z \widehat{T}_{K} & O
\end{array}\right]
$$

To compute the finite zeros and Kronecker structure of the periodic system, we thus apply to the resulting lower order subpencils a general algorithm to compute the eigenvalues and the Kronecker structure of a system matrix of a particular descriptor system [16].

The proposed algorithm to compute zeros can be applied to compute the poles as well by defining

$$
S_{i}:=A_{i}, \quad T_{i}:=I_{n_{i+1}} .
$$

In a similar way, with

$$
S_{i}:=\left[\begin{array}{ll}
A_{i} & B_{i}
\end{array}\right], \quad T_{i}:=\left[\begin{array}{ll}
I_{n_{i+1}} & O
\end{array}\right]
$$

or

$$
S_{i}:=\left[\begin{array}{c}
A_{i} \\
C_{i}
\end{array}\right], \quad T_{i}:=\left[\begin{array}{c}
I_{n_{i+1}} \\
O
\end{array}\right]
$$

the zeros algorithm can be used to compute the input decoupling and output decoupling zeros, respectively [11].

The zeros algorithm can be extended without any modification to compute the zeros of a periodic descriptor system

$$
\begin{aligned}
E_{k} x(k+1) & =A_{k} x(k)+B_{k} u(k) \\
y(k) & =C_{k} x(k)+D_{k} u(k)
\end{aligned}
$$

where $E_{k} \in \mathrm{R}^{n_{k+1} \times n_{k+1}}$ and is possibly non-singular. In this case the building blocks of the system pencil (8) are

$$
S_{i}:=\left[\begin{array}{cc}
A_{i} & B_{i} \\
C_{i} & D_{i}
\end{array}\right], \quad T_{i}:=\left[\begin{array}{cc}
E_{i} & O \\
O & O
\end{array}\right]
$$

For the computations of poles of the periodic descriptor system (9) these blocks are redefined as

$$
S_{i}:=A_{i}, \quad T_{i}:=E_{i}
$$

For the descriptor case, the resulting algorithm for computation of poles generalizes the procedure of [20] to the case of time-varying state dimensions. 


\section{Algorithmic aspects}

The reduction of $S(z)$ can be done by computing successively $K-1$ rank revealing QR decompositions (with column pivoting) of $\left(\nu_{i}+n_{i+1}+2 p\right) \times\left(n_{i+1}+m\right)$ matrices $\left(\nu_{i} \geq n_{i}\right)$ and applying the transformation to two sub-blocks of dimensions $\left.\left(\nu_{i}+n_{i+1}+2 p\right) \times n_{i+1}\right)$ and $\left(\nu_{i}+n_{i+1}+2 p\right) \times\left(n_{i+2}+m\right)$. Assuming constant dimensions $n_{i}=n, p \leq m$ and generic ranks (i.e., $\nu_{i}=n_{i}$ ), the reduction step has a computational complexity of $O((K-1)(n+p)(n+m) n)$. Since the last step, the computation of zeros of the reduced pencil, has a complexity of $O((n+p)(n+m) n)$, it follows that the overall computational complexity of the proposed approach corresponds to what is expected for a satisfactory algorithm for periodic systems. In fact, when this approach is employed to compute the poles, it is even more efficient than the standard algorithm based on the periodic real Schur form [3]. This is why, the proposed algorithm belong to the family of fast algorithms [20], being more efficient than an algorithm based on eigenvalue computation (if this is applicable, as for example, in the case of invertible $\mathcal{D}$ ).

Since both the reduction step and the subsequent, zeros computation step, are based exclusively on orthogonal transformations, the proposed algorithm to compute zeros is backward stable, and thus the computed zeros are exact for a slightly perturbed system pencil. However, since the structure of the perturbed pencil is not preserved in the reduction, we can not say that the computed zeros are exact for a slightly perturbed original system. In spite of this weaker type of stability, the proposed algorithm is the first numerically reliable procedure able to compute zeros of a periodic system with an acceptable computational effort.

\section{Examples}

Example 1. Consider the 2-periodic single-input single-output system described by the following matrices

$$
\begin{aligned}
& A_{1}=\left[\begin{array}{c}
0 \\
0.5
\end{array}\right], \quad B_{1}=\left[\begin{array}{l}
1 \\
0
\end{array}\right], C_{1}=1, \quad D_{1}=0 \\
& A_{2}=\left[\begin{array}{ll}
0 & 0.5
\end{array}\right], B_{2}=1, \quad C_{2}=\left[\begin{array}{ll}
1 & 0
\end{array}\right], D_{2}=0
\end{aligned}
$$

where the state-space dimensions are $n_{1}=1, n_{2}=2$. This system has no input or output decoupling zeros, thus is minimal (i.e., reachable and observable). The system has a pole at $\rho=0.25$ and a zero at $\mu=\infty$.

Example 2. Consider the 3-periodic single-input single-output system

$$
\begin{array}{ll}
A_{1}=\left[\begin{array}{ll}
0 & 0.9 \\
0 & 0
\end{array}\right], & B_{1}=\left[\begin{array}{l}
3 \\
0
\end{array}\right], C_{1}=\left[\begin{array}{ll}
0 & 1
\end{array}\right], D_{1}=1 \\
A_{2}=\left[\begin{array}{ll}
1 & 2 \\
0 & 0
\end{array}\right], & B_{2}=\left[\begin{array}{l}
0 \\
1
\end{array}\right], C_{2}=\left[\begin{array}{ll}
2 & 4
\end{array}\right], D_{2}=3 \\
A_{3}=\left[\begin{array}{ll}
0 & 0 \\
1 & 4
\end{array}\right], & B_{3}=\left[\begin{array}{l}
0 \\
1
\end{array}\right], C_{3}=\left[\begin{array}{ll}
3 & 1
\end{array}\right], D_{3}=1
\end{array}
$$

with state dimensions $n_{1}=n_{2}=n_{3}=2$. This system is not minimal, having an input-decoupling zero at $\nu=0$. Therefore, the computed poles $\rho_{1}=1, \rho_{2}=0$ and zeros $\mu_{1}=8, \mu_{2}=0$ also include the uncontrollable pole $\nu=0$.

Example 3. Consider a discrete-time periodic system originating from a continuous-time periodic model of a spacecraft pointing and attitude system described in [18]. This system has state, input and output dimensions $n=4, m=1, p=2$, respectively. The discretized system for different sampling periods $K$ has been used in [24] to compare periodic output feedback controllers designed for this system. The periodic system for $K=120$ has a zero at $\mu=\infty$ and has poles at

$$
\begin{aligned}
& \rho_{1}=0.7626+0.6469 i \\
& \rho_{2}=0.7626-0.6469 i \\
& \rho_{3}=0.9942+0.1077 i \\
& \rho_{4}=0.9942-0.1077 i
\end{aligned}
$$

Note that the order of the stacked lifted system is 480 . Although the direct application of the zeros algorithm of [16] to this system is still feasible, it is certainly too expensive to solve this problem.

In the following table, computational times are given to determine the zeros of the associated TFM for different values of $K$. The values for $T_{\text {fast }}$ represent computational times for the proposed fast method, while the values for $T_{\text {lifted }}$ are the times when applying the algorithm of [16] directly to the stacked lifted system. The computations have been done on a $866 \mathrm{MHz} \mathrm{PC}$ running Matlab 6.1 under Windows ME.

\begin{tabular}{c|rrrr}
$K$ & 40 & 80 & 120 & 240 \\
\hline$T_{\text {fast }}(\mathrm{sec})$ & 0.05 & 0.06 & 0.11 & 0.17 \\
$T_{\text {lifted }}(\mathrm{sec})$ & 0.33 & 2.31 & 7.74 & 61.46
\end{tabular}

Table 1: Computational times for zeros determination

It is easy to see that the computational time for the fast method varies almost linearly with $K$, and this confirms our claim for a computational complexity of $O\left(K^{3}\right)$ of the proposed approach. In contrast, when applying the algorithm of [16] to the stacked lifted system, the resulting times clearly indicates a computational complexity of $O\left(K^{3} n^{3}\right)$. 


\section{Conclusion}

In this paper we presented a numerically backward stable algorithm to compute the generalized eigenstructure of a stacked system matrix of a periodic system. This algorithm can be applied to find the zeros, poles and decoupling zeros of the system matrix and the left and right null space structures of the corresponding lifted transfer function. The algorithm exploits the block cyclic structure of the pencil to yield a complexity which is linear in the period $K$ and cubic in the dimension $n=\max _{i} n_{i}$ of the blocks. The method also works for matrices of varying dimension and can easily be extended to handle periodic systems in generalized state space form.

\section{References}

[1] P. Benner and R. Byers. Evaluating products of matrix pencils and collapsing matrix products. Lin. Alg. \& Appl., 8:357-380, 2001.

[2] S. Bittanti and P. Colaneri. Analysis of discretetime linear periodic systems. In C. T. Leondes (Ed.), Digital Control and Signal Processing Systems and Techniques, vol. 78 of Control and Dynamics Systems, pp. 313-339. Academic Press, 1996.

[3] A. W. Bojanczyk, G. Golub, and P. Van Dooren. The periodic Schur decomposition. Algorithms and applications. In F. T. Luk (Ed.), Proceedings SPIE Conference, vol. 1770, pp. 31-42, July 1992.

[4] P. Bolzern, P. Colaneri, and R. Scattolini. Zeros of discrete-time linear periodic systems. IEEE Trans. Autom. Control, 31:1057-1058, 1986.

[5] P. Colaneri and S. Longhi. The realization problem for linear periodic systems. Automatica, 31:775779, 1995.

[6] A. Emami-Naeini and P. M. Van Dooren. Computation of zeros of linear multivariable systems. Automatica, 18:415-430, 1982.

[7] D. S. Flamm. A new shift-invariant representation of periodic linear systems. Systems \& Control Lett., 17:9-14, 1991.

[8] I. Gohberg, M. A. Kaashoek, and J. Kos. Classification of linear periodic difference equations under periodic or kinematic similarity. SIAM J. Matrix Anal. Appl., 21:481-507, 1999.

[9] I. Gohberg, M. A. Kaashoek, and L. Lerer. Minimality and realization of discrete time-varying systems. Operator Theory: Advances and Applications, 56:261296, 1992.

[10] O. M. Grasselli and S. Longhi. Zeros and poles of linear periodic discrete-time systems. Circuits, Systems and Signal Processing, 7:361-380, 1988.
[11] O. M. Grasselli and S. Longhi. Finite zero structure of linear periodic discrete-time systems. Int. J. Systems Sci., 22:1785-1806, 1991.

[12] O. M. Grasselli and S. Longhi. Pole-placement for nonreachable periodic discrete-time systems. Math. Control Signals Syst., 4:439-455, 1991.

[13] D. G. Luenberger. Boundary recursion for descriptor variable systems. IEEE Trans. Autom. Control, 34:287-292, 1989.

[14] A. MacFarlane and N. Karcanias. Poles and zeros of linear multivariable systems: a survey of the algebraic, geometric, and complex-variable theory. Int. J. Control, 24:33-74, 1976.

[15] R. A. Meyer and C. S. Burrus. A unified analysis of multirate and periodically time-varying digital filters. IEEE Trans. Circuits and Systems, 22:162-168, 1975.

[16] P. Misra, P. Van Dooren, and A. Varga. Computation of structural invariants of generalized state-space systems. Automatica, 30:1921-1936, 1994.

[17] B. Park and E. I. Verriest. Canonical forms for discrete-time periodically time varying systems and a control application. Proc. of CDC'89, Tampa, pp. 1220-1225, 1989.

[18] M. E. Pittelkau. Optimal periodic control for spacecraft pointing and attitude determination. J. of Guidance, Control, and Dynamics, 16:1078-1084, 1993.

[19] F. Svaricek. Computation of the structural invariants of linear multivariable systems with an extended version of the program ZEROS. Systems \& Control Lett., 6:261-266, 1995.

[20] P. Van Dooren. Two point boundary value and periodic eigenvalue problems. Proc. CACSD'99 Symposium, Kohala Coast, Hawaii, 1999.

[21] A. Varga. Balancing related methods for minimal realization of periodic systems. Systems \& Control Lett., 36:339-349, 1999.

[22] A. Varga. Computation of transfer functions matrices of periodic systems. Proc. of CDC'2002, Las Vegas, Nevada, 2002.

[23] A. Varga and P. Van Dooren. Computational methods for periodic systems - an overview. Proc. of IFAC Workshop on Periodic Control Systems, Como, Italy, pp. 171-176, 2001.

[24] A. Varga and S. Pieters. Gradient-based approach to solve optimal periodic output feedback control problems. Automatica, 34:477-481, 1998. 